Assurances et gestion des risques

Insurance and Risk Management

ASSURANCES

\title{
Théorie des ensembles flous : une application à l'assurance indicielle au Burkina Faso
}

\section{Abel Tiemtore}

Volume 85, numéro 3-4, décembre 2018

URI : https://id.erudit.org/iderudit/1056944ar

DOI : https://doi.org/10.7202/1056944ar

Aller au sommaire du numéro

\section{Éditeur(s)}

Faculté des sciences de l'administration, Université Laval

ISSN

1705-7299 (imprimé)

2371-4913 (numérique)

Découvrir la revue

Citer cet article

Tiemtore, A. (2018). Théorie des ensembles flous : une application à l'assurance indicielle au Burkina Faso. Assurances et gestion des risques / Insurance and Risk Management, 85(3-4), 173-192. https://doi.org/10.7202/1056944ar

\section{Résumé de l'article}

L'assurance indicielle, considérée dès son introduction dans les pays en développement comme un puissant outil de stabilisation des revenus des paysans et de réduction de la pauvreté, a très vite désenchanté ses promoteurs. L'évolution de la demande de cette assurance est particulièrement décevante dans la majeure partie des pays qui l'ont expérimenté. Au Burkina Faso, on constate une forte baisse des adhésions et partant des superficies et des sommes assurées. La principale raison évoquée par les producteurs est l'absence d'indemnisations ou le très faible niveau d'indemnisation en cas de perte de production (risque de base). La présente étude propose l'utilisation des techniques de classification floue qui autorise l'appartenance partielle à des classes à indemniser pour réduire le risque de base et augmenter l'attractivité de l'assurance indicielle au sein des producteurs de coton au Burkina Faso. Nous avons montré que l'application de la technique de classification floue augmente significativement la probabilité d'être indemnisé (de plus de trois fois par rapport à son niveau actuel) mais aussi la prime de l'assurance. Toutefois selon Elabed et Carter (2014a), les producteurs seraient prêts à payer des sommes substantielles pour atténuer ou éliminer totalement le risque de base.
Tous droits réservés $\odot$ Faculté des sciences de l'administration, Université Laval, 2019
Ce document est protégé par la loi sur le droit d'auteur. L'utilisation des services d'Érudit (y compris la reproduction) est assujettie à sa politique d'utilisation que vous pouvez consulter en ligne.

https://apropos.erudit.org/fr/usagers/politique-dutilisation/ 


\title{
THÉORIE DES ENSEMBLES FLOUS: UNE APPLICATION À L'ASSURANCE INDICIELLE AU BURKINA FASO
}

\author{
Abel TIEMTORE ${ }^{1}$
}

\section{RÉSUMÉ}

L'assurance indicielle, considérée dès son introduction dans les pays en développement comme un puissant outil de stabilisation des revenus des paysans et de réduction de la pauvreté, a très vite désenchanté ses promoteurs. L'évolution de la demande de cette assurance est particulièrement décevante dans la majeure partie des pays qui l'ont expérimenté. Au Burkina Faso, on constate une forte baisse des adhésions et partant des superficies et des sommes assurées. La principale raison évoquée par les producteurs est l'absence d'indemnisations ou le très faible niveau d'indemnisation en cas de perte de production (risque de base). La présente étude propose l'utilisation des techniques de classification floue qui autorise l'appartenance partielle à des classes à indemniser pour réduire le risque de base et augmenter l'attractivité de l'assurance indicielle au sein des producteurs de coton au Burkina Faso. Nous avons montré que l'application de la technique de classification floue augmente significativement la probabilité d'être indemnisé (de plus de trois fois par rapport à son niveau actuel) mais aussi la prime de l'assurance. Toutefois selon Elabed et Carter (2014a), les producteurs seraient prêts à payer des sommes substantielles pour atténuer ou éliminer totalement le risque de base.

Mots clés: assurance indicielle, théorie des ensembles flous, classification floue, classification dure (classique), fuzzyfication

\section{ABSTRACT}

Index insurance, considered as a powerful tool for stabilizing farmers' incomes and reducing poverty at its introducing in developing countries, soon disenchanted its promoters. The evolution of demand for this insurance is particularly disappointing in most of the countries that have implemented it. In Burkina Faso, there has been a sharp decline in adhesion and hence in amounts insured. The 
main reason given by the producers is the lack of payment or the very low level of insurance payout in case of loss of production (basic risk). This study proposes the use of fuzzy classification techniques that allow partial membership of classes to be eligible for a payment to reduce the basic risk and increase the attractiveness of index insurance among cotton producers in Burkina Faso. We have shown that the application of the fuzzy classification technique significantly increases the probability of being compensated (more than three times compared to its current level) but also the premium of the insurance. However, according to Elabed and Carter (2014a), producers would be willing to pay substantial sums to mitigate or completely eliminate the basic risk.

Keywords: index insurance, fuzzy set theory, fuzzy classification, crisp classification (classical), fuzzyfication.

\section{INTRODUCTION}

L'agriculture est considérée comme la pierre angulaire des économies africaines selon la Banque Mondiale (2015). Toutefois les catastrophes naturelles liées au changement climatique ont un impact majeur sur les petits exploitants agricoles de la région africaine (FAO, 2015). Dans une telle situation, l'assurance indicielle est considérée comme un outil prometteur pour améliorer la résilience des agriculteurs et les prémunir contre les risques de production. Cependant, en dépit des nombreux avantages qu'elle présente sur le plan théorique, l'assurance indicielle reste confrontée à des défis d'évolutivité et de durabilité (Elabed, 2014). Au Burkina Faso, on constate une forte baisse des adhésions et partant des superficies et des sommes assurées (Planet Guarante, 2017). La principale raison est que les produits de l'assurance agricole indicielle sont exposés à un risque de base élevé. Skees (2008) et Clarke (2011a) considèrent le risque de base comme le déterminant principal d'une faible demande de l'assurance indicielle. Selon Carter et al. (2015), la demande d'assurance indicielle dépend principalement de la qualité de la conception des contrats. En effet, un rapport de OXFAM Burkina (2016) indique que les principales raisons avancées par les producteurs qui abandonnent l'assurance indicielle sont le manque de moyens financiers mais surtout l'absence d'indemnisations ou le très faible niveau d'indemnisation alors que les producteurs pensent avoir perdu une partie de leur récolte. Toutefois les effets potentiels du risque de base peuvent être considérablement réduits en concevant de meilleurs indices, avec une plus forte granularité et plus étroitement liés aux 
pertes des agriculteurs; ce qui revient à améliorer le degré de corrélation entre les paiements d'assurance perçus par les agriculteurs et les chocs de production.

Une des principales composantes du risque de base est le risque lié à la conception du produit notamment la fixation de seuils rigides de déclenchement des différents taux d'indemnisation. L'assurance indicielle rendement offerte aux cotonculteurs burkinabè définit pour chaque catégorie de producteurs trois niveaux critiques (ou seuils) de déclenchement des différents taux d'indemnisations. Ainsi chaque producteur, à travers le groupement de producteurs de coton (GPC) auquel il appartient, est classé de façon exclusive dans une des quatre classes définies par l'indice. Le passage d'une classe à une autre se fait de façon abrupte (pour une différence de quelques grammes à l'hectare, deux producteurs se retrouvent dans des classes différentes et reçoivent des indemnités différentes pouvant aller du simple au triple). Il est donc question dans cette étude, d'assouplir cette division abrupte des producteurs en prenant en compte le fait que le passage d'une classe à une autre se fait de manière graduelle. Une façon de prendre en compte ce fait est d'introduire le concept d'appartenance partielle qui autorise une transition continue entre les différentes classes. La notion d'appartenance partielle a été introduite à travers la théorie des ensembles flous développée par Zadeh en 1965. Elle autorise une appartenance partielle d'un élément à un ensemble. Selon Lemaire (1990) la théorie des ensembles flous fournit un meilleur cadre que la théorie des probabilités pour la modélisation des phénomènes qui ont une imprécision inhérente. Elle permet de prendre en compte dans le processus de sélection et d'évaluation du risque à assurer des situations caractérisées par une incertitude qui ne peut être correctement décrite par la probabilité (Derrig \& Ostaszewski 1994). L'hypothèse principale de cette recherche est que la fuzzyfication contribue à une réduction significative du risque de base en assurance indicielle.

Le reste du texte se présente de la manière suivante: dans la première partie nous présentons l'assurance indicielle rendement coton au Burkina Faso, la deuxième partie présente une brève revue de littérature sur les applications de la logique floue à l'assurance, la troisième partie présente de façon succincte la théorie des ensembles flous et l'algorithme d'application au secteur de l'assurance. Enfin, la quatrième partie, est consacrée aux résultats de l'application de la théorie des ensembles flous à la classification des cotonculteurs dans les classes de risques prédéfinis. 


\section{L'ASSURANCE INDICIELLE RENDEMENT COTON AU BURKINA FASO}

Les agriculteurs burkinabè sont confrontés à de nombreuses sources de risques, en particulier la sécheresse et les inondations, mais aussi des chocs localisés tels que les ravageurs, les maladies, les animaux ou d'autres chocs de production (perte de main-d'ouvre) (Barré et al. 2016). La culture du coton est hautement profitable mais particulièrement risquée du fait de la sensibilité des rendements aux variables climatiques et de l'absence de mécanismes d'assurance au profit des agriculteurs (Stoeffler et al. 2016). Le secteur du coton au Burkina Faso est dominé par des sociétés parapubliques qui ont des monopoles locaux dans de grandes régions. L'expérimentation de l'assurance indicielle est faite dans la zone de la SOFITEX (Société burkinabè des fibres et textiles) qui représente $80 \%$ de la production nationale.

L'assurance indicielle coton offerte aux producteurs burkinabè repose sur un mécanisme à double seuils basé sur le rendement. Elle s'appuie sur l'organisation très structurée du secteur du coton dans le pays. L'assurance indicielle rendement du coton au Burkina Faso est conçue sur la base des données collectées par la Sofitex. La Sofitex achète l'ensemble de la production aux groupements paysans (GPC). Chaque producteur de coton doit appartenir à un GPC de dix à quarante membres (mais parfois jusqu'à quatre-vingts membres de facto). La Sofitex fournit tous les intrants à crédit (semences, engrais, pesticides, etc.) en utilisant la production cotonnière du groupe comme garantie. A travers ses agents techniques sur le terrain, la SOFITEX accompagne les producteurs depuis les semis jusqu'à la récolte et effectue la pesée de la production individuelle des membres des différents GPC. Ainsi elle dispose de données réelles sur les rendements individuels des producteurs et les rendements moyens des GPC. Les GPC ont été regroupés en quatre catégories selon leur rendement historique moyen: la distribution du rendement a été estimée pour chaque catégorie et chaque catégorie s'est vue offrir un contrat différent selon l'historique de ses rendements.

L'assurance fournit trois niveaux de paiement. Lorsque les rende-

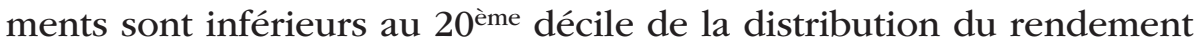
(un événement sur cinq ans), les agriculteurs reçoivent un "petit paiement" de 11200 FCFA par hectare assuré. Ce paiement a été conçu pour correspondre à la valeur de la prime d'assurance, il assure que la prime est remboursée aux agriculteurs en cas de choc léger. Lorsque les rendements sont inférieurs au $8^{\text {ème }}$ décile de la distribution du rendement, l'assurance offre un "paiement moyen» de 34000 FCFA. Enfin, 
dans le cas de rendements inférieurs au 4ème décile de la distribution (un événement sur 25 ans), les agriculteurs reçoivent un "gros paiement" de 90000 FCFA par hectare, ce qui correspond approximativement à la valeur du prêt sur les intrants. Les rendements utilisés pour le calcul des paiements de l'assurance sont ceux pesés par les agents de la Sofitex. En tant que tel, il couvre tous les types de chocs covariants, ce qui constitue un net avantage par rapport aux produits de l'assurance indicielle basés sur un indice pluviométrique par exemple et ne couvre que les chocs liés à la pluviométrie.

À l'instar du produit mis en place au Mali, l'assurance indicielle Burkinabè a un double mécanisme de déclenchement des indemnisations (Elabed et al., 2013). Cela signifie que les agriculteurs reçoivent des paiements lorsque deux conditions sont respectées simultanément.

Premièrement, le rendement du GPC doit être inférieur à un seuil donné correspondant au rendement de sa catégorie (par exemple: rendements inférieurs à $800 \mathrm{~kg} / \mathrm{ha}$ ). Deuxièmement, les autres GPC dans le voisinage du GPC assuré doivent également avoir des rendements assez bas: il y a aussi un seuil de "voisinage", qui est plus élevé que le seuil du GPC assuré (par ex. être inférieur à $1000 \mathrm{~kg} / \mathrm{ha}$ ). Cette condition de voisinage a été introduite pour éviter les problèmes potentiels d'aléa moral. En effet, comme les agriculteurs d'un groupe vivent dans le même village et sont généralement membres de la même famille, du même groupe ethnique ou de la même communauté religieuse, il est probable observer une certaine coordination dans les décisions de production au sein du groupe. La condition sur le voisinage empêche une telle coordination en veillant à ce que les rendements ne soient pas particulièrement bas dans certains groupes de la région. Cependant, cela introduit un certain risque de base au niveau du groupe (Stoeffler et al. 2016).

La prime commerciale est d'environ 11200 FCFA par hectare, soit au-dessus de la prime actuariellement équitable d'au moins $75 \%$ (Barré et al. 2016).

\begin{tabular}{|c|c|c|c|c|}
\hline CAMPAGNE & 2013/2014 & $2014 / 2015$ & 2015/2016 & 2016/2017 \\
\hline Superficie couverte (ha) & 2331 & 4448 & 1449 & 1540 \\
\hline Montant total assuré (FCFA) & 233100000 & 444825000 & 144900000 & 116550000 \\
\hline Prime due à l'assureur (FCFA) & 26107200 & 49820400 & 16228800 & 13053600 \\
\hline Indemnisation (FCFA) & 12825000 & 52984000 & 35554200 & - \\
\hline Rapport Indemnisation/prime & 0,4912 & 1,0635 & 2,1908 & - \\
\hline
\end{tabular}

Source : Planet Guarantee Burkina Faso 
Sur l'ensemble des trois campagnes agricoles consécutives qui ont suivi le lancement de l'assurance rendement-coton, le montant des indemnisations s'élève à $110 \%$ du montant des primes collectées sur la même période. L'analyse des résultats fait ressortir une forte baisse des adhésions et partant des superficies et sommes assurées. Les principales raisons avancées par les producteurs selon le rapport de OXFAM Burkina (2016) sont le manque de moyens financiers mais surtout l'absence d'indemnisations ou le très faible niveau d'indemnisation alors que les producteurs pensent avoir perdu une partie de leur récolte. Etant donné que la principale cause de démotivation des producteurs se situe au niveau de l'indemnisation donc de leur classification dans les différentes classes, l'extension de l'assurance agricole au Burkina Faso passe nécessairement par l'amélioration de la classification des producteurs pour mieux refléter les chocs de production qu'ils auront connus.

La classification classique utilisée consiste à ranger les producteurs dans les différentes classes de façon exclusive, un producteur appartient à une et une seule classe. Cette classification présente de nombreuses insuffisances la rendant plus ou moins aléatoire. Premièrement elle est basée sur le rendement moyen du groupe (GPC) et non sur le rendement individuel du producteur d'où une source de risque de base liée à l'hétérogénéité des rendements à l'intérieur du GPC. Deuxièmement, elle dépend de la catégorie dans laquelle est classée le GPC, d'où une source de risque de base liée à l'hétérogénéité à l'intérieur de la catégorie. Enfin, elle dépend du rendement moyen des GPC géographiquement voisins; c'est-à-dire des GPC situés dans un espace géographique tel qu'ils subissent des conditions climatiques similaires. Cette dernière condition introduit une autre source de risque de base liée à l'hétérogénéité de l'espace considéré et aux risques idiosyncratiques pouvant affecter des individus d'un GPC donné sans toucher ceux des autres GPC voisins.

\section{ReVUe DE LITTÉRATURE}

Le domaine de la science actuarielle a rejoint tardivement les domaines d'application de la méthodologie des ensembles flous (Derrig et Ostaszewski, 1999). Introduite par Zadeh en 1965, la théorie des ensembles flous a vite suscité un grand intérêt auprès des chercheurs, on notait déjà en 1980 plus de 2500 articles scientifiques publiés dans ce domaine (Chen et al., 1980). La première application de la méthodologie des ensembles flous à la science actuarielle est de Dewit (1982) 
qui montre que le processus de formulation du contrat d'assurance, c'est-à-dire le processus de sélection et d'évaluation des risques à assurer, est lourdement affecté d'incertitude que la probabilité peut ne pas pouvoir décrire de façon satisfaisante. Après l'article de Dewit (19982), Earbach et al. (1987) combinent la méthodologie des ensembles flous avec d'autres techniques pour développer un prototype d'assurance vie appelé "zeno" au profit d'une compagnie d'assurance au Canada. Lemaire (1990) prolonge les travaux de Dewit (19982) en suggérant l'utilisation de la logique floue dans la formulation du contrat d'assurance en générale et particulièrement dans les calculs de la prime et des réserves à effectuer. Young (1993) publie un algorithme spécifique à l'écriture des contrats d'assurance santé destinés à des groupes d'individus en utilisant la méthode floue. Ebanks et al. (1992) utilisent la mesure floue dans la classification des risques. Ostaszewski (1993) montre les limites de la classification traditionnelle en assurance santé, selon cette méthode un individu peut se voir classé dans un groupe de personnes à risque pour seulement quelques grammes de plus lorsque le poids est utilisé comme indicateur de santé. Il montre que la classification floue, en attribuant des degrés d'appartenance aux différentes classes de risque situe plus l'individu dans un groupe intermédiaire reflétant mieux son niveau de risque. Derrig et Ostaszewski (1995) utilisent le regroupement flou dans la classification des risques et des indemnisations, ils appliquent la méthodologie floue dans l'affectation des véhicules automobiles aux différents territoires dans les Massachussetts. Ils conclurent que la classification floue décrit mieux la situation des villes situées à cheval entre deux territoires. Cummins et Derrig (1997) derivent des primes d'assurances floues comme une généralisation des primes du modèle probabiliste. Verral et Yakoubov (1999) utilisent la théorie des ensembles flous pour analyser la classification selon l'âge en assurance générale; ils montrent qu'un mauvais regroupement selon l'age peut conduire à un biais dans la tarification et les indemnisations au niveau de l'assureur. Andrés-Sanchez (2014) développe des expressions pour quantifier les provisions des dédommagements utilisant la logique floue.

\section{THÉORIE DES ENSEMBLES FLOUS}

\subsection{Définition d'un sous-ensemble flou}

Dans la logique booléenne, un élément appartient ou n'appartient pas à un ensemble donné: c'est le "tout ou rien". Zadeh (1965) constate que le plus souvent, les objets rencontrés dans le monde réel n'ont pas 
de critères précis d'appartenance. Il va tenter alors de sortir de cette logique booléenne en introduisant la notion d'appartenance pondérée. Il définit l'ensemble flou comme une classe d'objets avec un continuum de degrés d'appartenance à cette classe. Un tel ensemble est caractérisé par une fonction d'appartenance qui associe à chaque objet un degré d'appartenance compris entre zéro et un.

Soit $\mathrm{X}$ un ensemble de référence et soit $x$ un élément quelconque de $\mathrm{X}$. Un ensemble flou A de X est défini comme l'ensemble des couples:

$$
\mathrm{A}=\left\{\left(x, \mu_{\mathrm{A}}(x)\right), x \in \mathrm{X}\right\}
$$

avec

$$
\mu_{\mathrm{A}}: \mathrm{X} \rightarrow[0,1]
$$

Ainsi, un ensemble flou A de $\mathrm{X}$ est caractérisé par une fonction d'appartenance qui associe, à chaque élément $x$ de $\mathrm{X}$ un réel dans l'intervalle $[0,1] ; \mu_{\mathrm{A}}(x)$ représente le degré d'appartenance de $x$ à $\mathrm{A}$. Ainsi, plus la valeur de $\mu_{\mathrm{A}}(x)$ est proche de l'unité et plus le degré d'appartenance de $x$ à A est élevé.

Si on a:

$\mu_{\mathrm{A}}: \mathrm{X} \rightarrow\{0,1\}$ on retrouve le cas booléen, soit $x$ appartient à $\mathrm{A}\left(\mu_{\mathrm{A}}=1\right)$ soit il n'appartient pas à $\mathrm{A}\left(\mu_{\mathrm{A}}=0\right)$.

\subsection{Propriétés des ensembles flous}

Comme la théorie des ensembles flous est une extension de la théorie des ensembles classiques, les propriétés existantes des ensembles classiques doivent être étendues aux ensembles flous et de nouvelles propriétés introduites.

Définition 1: L'ensemble flou est considéré comme vide si les degrés d'appartenance de tous les éléments de l'univers sont tous égaux à zéro.

$$
\mathrm{A}=\varnothing \Leftrightarrow \mu_{\mathrm{A}}(x)=0, \quad \forall x \in \mathrm{X}
$$

Deux ensembles flous sont égaux si leurs degrés d'appartenance sont égaux pour tous les éléments de l'ensemble de référence, c'est-à-dire si les deux ensembles flous ont la même fonction d'appartenance. 
Définition 2: Deux ensembles flous A et B, définis sur le même ensemble de référence $\mathrm{X}$ sont égaux si:

$$
\mathrm{A}=\mathrm{B} \Leftrightarrow \mu_{\mathrm{A}}(x)=\mu_{\mathrm{B}}(x), \quad \forall x \in \mathrm{X}
$$

Un ensemble flou A est inclus dans un ensemble flou B si les degrés d'appartenance de A sont inférieurs ou égaux aux degrés d'appartenance de $\mathrm{B}$ pour tous les éléments de l'univers.

Définition 3: Soient A et B deux ensembles flous définis sur X, A est inclus dans B si:

$$
\mathrm{A} \subset \mathrm{B} \Leftrightarrow \mu_{\mathrm{A}}(x) \leq \mu_{\mathrm{B}}(x), \quad \forall x \in \mathrm{X}
$$

La cardinalité d'un ensemble ordinaire est égale au nombre d'éléments qu'il contient. Dans un ensemble flou, les éléments peuvent avoir une appartenance partielle, donc la cardinalité est la somme des degrés d'appartenance des éléments de l'ensemble de référence. Il est également possible de dériver la cardinalité relative d'un ensemble flou en divisant la cardinalité de l'ensemble flou par la cardinalité de l'univers. La cardinalité relative permet de comparer des ensembles flous s'ils sont basés sur le même univers.

Définition 4: La cardinalité et la cardinalité relative d'un ensemble flou A, défini sur un univers fini $\mathrm{X}$, sont ainsi définies:

$$
\begin{aligned}
& \operatorname{Card}(\mathrm{A})=|\mathrm{A}|=\sum_{x \in \mathrm{X}} \mu_{\mathrm{A}}(x) \\
& \operatorname{RelCard}(\mathrm{A})=\|\mathrm{A}\|=\frac{|\mathrm{A}|}{|\mathrm{X}|}
\end{aligned}
$$

Un ensemble flou est convexe si un point situé entre deux autres points a un degré d'appartenance supérieur au degré d'appartenance minimum de ces points.

Définition 5: Un ensemble flou A défini sur un ensemble de référence X est convexe si

$$
\forall x, y \in \mathrm{X}, \forall \lambda \in[0,1]: \mu_{\mathrm{A}}[\lambda x+(1-\lambda y)] \geq \min \left[\mu_{\mathrm{A}}(x), \mu_{\mathrm{A}}(y)\right]
$$

Le support d'un ensemble flou est le sous-ensemble net de l'univers où les degrés d'appartenance sont supérieurs à zéro. 
Définition 6: Le support d'un ensemble flou A défini sur un ensemble de référence $\mathrm{X}$ est un sous-ensemble $\mathrm{X}$ noté $\operatorname{Supp(A)}$ tel que:

$$
\operatorname{Supp}(\mathrm{A})=\left\{x \in \mathrm{X}, \quad \mu_{\mathrm{A}}(x)>0\right\}
$$

La $\alpha$-coupe, resp. la $\alpha$-coupe forte d'un ensemble flou est le sousensemble net de l'univers où les degrés d'appartenance sont supérieurs ou égaux, resp. supérieur à la valeur $\alpha$ spécifiée.

Définition 7: La $\alpha$-coupe (A $\alpha)$ et la $\alpha$-coupe forte ( $\left.\mathrm{A}^{\prime} \alpha\right)$ d'un ensemble flou définies sur un ensemble de référence $\mathrm{X}$ sont des sous-ensembles de $\mathrm{X}$ tels que:

$$
\begin{aligned}
& \mathrm{A}_{\alpha}=\left\{x \in \mathrm{X}, \quad \mu_{\mathrm{A}}(x) \geq \alpha, \alpha \in[0,1]\right\} \\
& \mathrm{A}_{\alpha}^{\prime}=\left\{x \in \mathrm{X}, \quad \mu_{\mathrm{A}}(x)>\alpha, \alpha \in[0,1]\right\}
\end{aligned}
$$

Définition 8: Le noyau d'un ensemble flou est le sous-ensemble net de l'univers où les degrés d'appartenance sont égaux à 1

$$
\operatorname{Kern}(\mathrm{A})=\left\{x \in \mathrm{X}, \quad \mu_{\mathrm{A}}(x)=1\right\}
$$

La hauteur d'un ensemble flou, noté h(A) est le degré d'appartenance maximum de tous les éléments de l'univers.

Définition 9: La hauteur d'un ensemble flou A défini sur un ensemble de référence borné $\mathrm{X}$ est ainsi définie:

$$
\mathrm{h}(\mathrm{A})=\operatorname{Max}_{x \in \mathrm{X}}\left(\mu_{\mathrm{A}}(x)\right)
$$

Un ensemble flou est dit normalisé si au moins un élément de l'univers a un degré d'appartenance égal à 1 .

Définition 10: Un ensemble flou A défini sur un ensemble de référence U est normalisé si et seulement si

$$
\exists x \in \mathrm{X}, \quad \mu_{\mathrm{A}}(x)=\mathrm{h}(\mathrm{A})=1
$$

La classification floue est une extension naturelle de la classification traditionnelle, de la même manière que les ensembles flous étendent les ensembles classiques. Dans une classification précise, chaque objet est assigné à exactement une classe, ce qui signifie que le degré d'appartenance de l'objet est 1 dans cette classe et 0 dans toutes les autres classes. L'appartenance des objets dans les classes est donc mutuellement exclusive. En revanche, une classification floue permet aux objets 
d'appartenir à plusieurs classes en même temps; de plus, chaque objet a des degrés d'appartenance qui expriment dans quelle mesure cet objet appartient aux différentes classes.

Le passage de la théorie des ensembles flous à la théorie des possibilités est simple. Le degré d'appartenance à un ensemble flou peut être interprété comme un facteur de certitude, un degré de vérité, un degré de satisfaction ou un degré de possibilité [Mukaidono, 2001]. En 1978, Zadeh a étendu la théorie des ensembles flous à une théorie des possibilités où les degrés d'appartenance sont considérés comme des degrés de possibilité.

Un concept central dans la théorie des possibilités est la distribution des possibilités qui est la contrepartie de la distribution de probabilités dans la théorie des probabilités. Une distribution de possibilité est un ensemble flou appelé restriction floue, qui agit comme une contrainte élastique, dont la fonction d'appartenance détermine la compatibilité ou la possibilité avec le concept de l'ensemble flou. Étant donné une distribution de possibilité, il est possible de calculer la possibilité d'un autre ensemble flou défini sur le même univers.

\subsection{L'algorithme des moyennes floues}

Nous utilisons une technique de classification floue (pattern recognition technique) développée par Bezdek (1981) et reprise par Derrig et Ostaszewski (...)

L'objectif est de repartir $n$ profils, où $n$ représente le nombre de producteurs de coton, chaque producteur ayant $p$ caractéristiques dans $c$, $2 \leq c \leq n$, classes homogènes. Ainsi les données se présentent sous forme d'une matrice $\mathrm{X}$ de dimensions $n \times p$ avec $\mathrm{X}=\left\{\mathrm{X}_{i}: i=1,2, \ldots, n\right\}$ et $\mathrm{X}_{i}=\left(x_{i 1}, x_{\mathrm{i} 2}, \ldots, x_{i p}\right)$.

Considérons deux producteurs $r$ et $s$, la décision de les regrouper dans une même classe est prise en considérant leur degré de similarité mesuré par:

$$
d\left(x_{r}, x_{s}\right)=\left\|\mathrm{X}_{r}-\mathrm{X}_{s}\right\|
$$

Le degré d'appartenance de chaque producteur aux différentes classes est obtenu en minimisant la somme pondérée des hétérogénéités intra-classes.

$$
z_{m}(\mathrm{M}, \mathrm{V})=\sum_{i=1}^{n} \sum_{k=1}^{c}\left(\mu_{i k}\right)^{m}\left\|\mathrm{X}_{r}-\mathrm{V}_{k}\right\|^{2}
$$


et $\mathrm{V}=\left\{\mathrm{V}_{k}: k=1,2, \ldots, c\right\}$ une matrice $c \times p$, avec $\mathrm{V}_{k}$ le centre de la classe $k$ à estimer. L'exposant $m$ détermine le poids accordé au dégré d'appartenance dans la procedure d'optimisation. Plus $m$ est grand et plus le poids accordé aux producteurs appartenant fortement à la classe est important. Si $m \rightarrow \infty$, la fonction d'appartenance tend vers la constante $1 / c$ indiquant le meme degré d'appartenance des différents producteurs aux différentes classes. Le minimum est atteint lorsque (Bezdek, 1981):

$$
\mathrm{V}_{k}=\frac{\sum_{i=1}^{n}\left(\mu_{i k}\right)^{m} \mathbf{X}_{i}}{\sum_{i=1}^{n}\left(\mu_{i k}\right)^{m}}
$$

avec

$$
\mu_{i k}=\frac{\left(\left\|\mathrm{X}_{r}-\mathrm{V}_{k}\right\|^{2}\right)^{-1 / m-1}}{\sum_{i=1}^{n}\left(\left\|\mathrm{X}_{i}-\mathrm{V}_{k}\right\|^{2}\right)^{-1 / m-1}}
$$

$\forall i=1,2, \ldots, n$; et $k=1,2, \ldots, c$.

La matrice $\mathrm{M}$ de dimensions $n \times c$ contenant les valeurs des fonctions d'appartenance aux classes floues

$$
\mathbf{M}=\left[\mu_{i k}\right]_{i=1, \ldots, n ; k=1, \ldots, c}
$$

est une c-partition floue si elle satisfait les conditions suivantes:

$$
\begin{gathered}
\sum_{k=1}^{c} \mu_{i k}=1 \quad \forall i=1,2, \ldots, n \\
0 \leq \sum_{i=1}^{n} \mu_{i k} \leq n \quad \forall k=1,2, \ldots, c
\end{gathered}
$$

L'algorithme de Bezdek (1981) consiste en quatre étapes:

Étape 1:

Choisir $c$, le nombre de classes; $c$ est tel que $n \leq c \leq n$. Dans notre situation $c=4$, le nombre de classes présentement définies par l'assureur' ${ }^{2}$.

Choisir le paramètre $m$, dans la pratique on choisit $m=2$ (Verall et Yakoubov, 1999).

Choisir une matrice $\mathrm{G}$ définie-positive de dimensions $p \times p$. Nous utilisons ici la matrice identité comme étant $G(G=I)$. Ce choix confère le même poids à toutes les caractéristiques du producteur. 
Définir la norme-G, comme $\mathrm{G}=\mathrm{I}$, la matrice identité, la norme est donnée par:

$$
\begin{aligned}
\left\|x_{i}-v_{k}\right\|_{\mathrm{G}} & =\sqrt{\left(x_{i}-\mathrm{v}_{k}\right)^{\prime}\left(x_{i}-v_{k}\right)} \\
& =\sqrt{\sum_{j=1}^{p} \sum_{l=1}^{p}\left(x_{i j}-v_{k l}\right)^{2}}
\end{aligned}
$$

Choisir la c-partition floue initiale

$$
\mathbf{M}^{0}=\left[\mu_{i k}^{0}\right]_{i=1, \ldots, n ; k=1, \ldots, c}
$$

Nous choisissons comme partition initiale la partition dure (classique) qui confère un degré d'appartenance égal à 1 si le rendement moyen courant classe la région dans la classe indiquée et 0 dans les autres classes. Ainsi la matrice $\mathbf{M}^{0}$ est constituée de 1 et de 0 , chaque ligne de la matrice comporte un seul 1 et le reste est formé de 0 .

Étape 2:

Calculer les centres des classes floues donnés par la formule suivante:

$$
v_{k}^{l}=\frac{\sum_{i=1}^{n}\left(\mu_{i k}^{l}\right)^{m} x_{i}}{\sum_{i=1}^{n}\left(\mu_{i k}^{l}\right)^{m}}
$$

pour $k=1, \ldots, c$.

Étape 3:

Calculer la nouvelle partition

$$
\mathbf{M}^{l+1}=\left[\mu_{i k}^{l+1}\right]_{i=1, \ldots, n ; k=1, \ldots, c}
$$

avec $\mu_{i k}^{l+1}=\frac{\left(\left\|\mathrm{X}_{i}-\mathrm{V}_{k}^{l}\right\|^{2}\right)^{-1 / m-1}}{\sum_{i=1}^{n}\left(\left\|\mathrm{X}_{i}-\mathrm{V}_{k}^{l}\right\|^{2}\right)^{-1 / m-1}}$

$$
\forall i=1, \ldots, n \text {; et } k=1, \ldots, c
$$

Si $\mathrm{X}_{i}=\mathrm{V}_{k}^{l}$, la formule (..) n'est pas utilisable. Dans ce cas on utilise:

$$
\mu_{i k}^{l+1}=\left\{\begin{array}{l}
1 \text { si } i=k \\
0 \text { si non }
\end{array} \text { pour } k=1, \ldots, c\right.
$$


Étape 4:

En utilisant l'extension de la norme-G à la norme matricielle ou d'autre norme matricielle, calculer:

$$
\Delta=\left\|\mathbf{M}^{l+1}-\mathbf{M}^{l}\right\|_{\mathrm{G}}
$$

Nous utilisons ici la norme simple, définie comme suit:

$$
\Delta=\sqrt{\sum_{k=1}^{c} \sum_{i=1}^{n}\left(\mu_{i k}^{l+1}-\mu_{i k}^{l}\right)^{2}}
$$

Si $\Delta>\varepsilon$, répéter les étapes 2,3 et 4 . Dans le cas contraire arrêter les itérations et considérer $\mathbf{M}^{l}=\left[\mu_{i k}^{l}\right]_{i=1, \ldots, n ; k=1, \ldots, c}$ comme la $c$-partition floue optimale.

Dans la présente étude $\varepsilon$ est fixé à 0.5 .

\section{LES DONNÉES}

Des études (Barron et al. 2003; Graef et Haigis 2001, Vischel et Lebel 2007) ont montré que la production agricole dépend fortement de la fréquence des pluies et de leur intensité. Les variables retenues dans la présente étude sont le rendement moyen du coton dans chaque région, la pluviométrie annuelle et le nombre de jours de pluie dans la région. Les données utilisées dans la présente étude viennent de deux sources. La production et les superficies cultivées du coton qui nous ont permis de calculer les rendements du coton proviennent de l'INSD (Institut National de la Statistique et de la Démographie), disponibles sur le site www.insd.bf dans la rubrique statistiques économiques. Elles concernent toutes les régions productrices de coton. La pluviométrie et le nombre de jours de pluies sont fournis par la direction nationale de la météorologie du Burkina Faso sur l'ensemble des régions du Burkina Faso par décade. Nous avons procédé à une simple sommation des données décadaires pour obtenir des données annuelles.

\section{LES RÉSULTATS}

L'application de la logique floue à la classification des différentes campagnes agricoles des principales régions cotonnières du Burkina Faso a produit les résultats suivants : 


\section{TABLEAU 1 Degrés d'appartenances moyens par région}

\begin{tabular}{|c|c|c|c|c|c|}
\hline \multicolumn{6}{|c|}{ RÉGION } \\
\hline \multicolumn{2}{|c|}{ Classification dure (probabilité) } & $4 \%$ & $4 \%$ & $12 \%$ & $80 \%$ \\
\hline \multirow{6}{*}{$\begin{array}{l}\text { Classification floue } \\
\text { (degré d'appartenance) }\end{array}$} & Cascades & $16,97 \%$ & $23,00 \%$ & $24,20 \%$ & $35,81 \%$ \\
\hline & Boucle du Mouhoun & $15,09 \%$ & $16,83 \%$ & $23,05 \%$ & $45,02 \%$ \\
\hline & Hauts Bassins & $23,67 \%$ & $27,41 \%$ & $22,66 \%$ & $26,24 \%$ \\
\hline & Sud-Ouest & $12,42 \%$ & $31,97 \%$ & $21,87 \%$ & $33,71 \%$ \\
\hline & Est & $23,11 \%$ & $24,78 \%$ & $29,77 \%$ & $22,32 \%$ \\
\hline & National & $18,25 \%$ & $24,80 \%$ & $24,31 \%$ & $32,62 \%$ \\
\hline
\end{tabular}

La classe 1 correspond à la classe des GPC ayant subi une perte totale de rendements, ils reçoivent une indemnisation de 90000 FCFA par hectare. La classe 2 est celle des GPC ayant subi une perte modérée de leurs rendements, ils perçoivent une indemnisation de 34000 FCFA par hectare, la classe 3 est celle des GPC ayant connu une faible baisse de rendement, ils perçoivent 11200 FCFA par hectare correspondant à un remboursement de leur prime d'assurance. Les GPC dans la classe quatre ne reçoivent aucune indemnisation.

Selon la classification dure présentement en vigueur au Burkina, un GPC moyen quelque soit sa catégorie ou sa zone agricole a $4 \%$ de chance d'être dans la classe un, $4 \%$ de chance d'être dans la classe deux, $12 \%$ de chance d'être dans la classe trois et $80 \%$ de chance d'être dans la classe quatre pour une campagne agricole donnée.

Selon la classification floue les possibilités d'appartenance aux différentes classes diffèrent d'une zone agricole à une autre. Sur le plan national, un GPC moyen a 18,25\% de chance (possibilité) d'être classé dans la classe un, $24,8 \%$ dans la classe deux, $24,3 \%$ dans la classe trois et $32,6 \%$ dans la classe quatre.

La classification floue donne plus de possibilité aux GPC d'être classés dans des classes indemnisables que la classification dure. Ainsi, en considérant la possibilité d'une appartenance partielle aux différentes classes, l'assurance indicielle agricole au Burkina Faso indemnisera en moyenne chaque GPC une fois tous les cinq ans et demi pour une situation de perte totale contrairement à la classification dure qui préconise ce type d'indemnisation une fois tous les 25 ans. 
Il est possible de jouer sur la fréquence de l'indemnisation en définissant un degré minimal d'appartenance pour bénéficier du paiement. En fixant un degré d'appartenance minimal de $20 \%$, soit $\alpha$-coupe $=0,20$, la fréquence de l'indemnisation totale tombe à une fois tous les 7 ans en moyenne. La classification dure correspond à une appartenance minimale de $51 \%$. Ainsi la classification floue tend vers la classification dure lorsque $\alpha$ augmente sous la condition que lorsque le degré d'appartenance à aucune classe ne vaut $\alpha$, le GPC est classé dans la classe quatre, c'est-à-dire celle des non indemnisables.

L'indemnisation forfaitaire par classe peut tenir compte du degré d'appartenance du GPC à la classe. La fuzzyfication du classement des GPC peut ainsi conduire à une indemnisation continue proportionnelle au degré d'appartenance de chaque GPC aux différentes classes indemnisables. L'avantage de la fuzzyfication est l'augmentation de la fréquence des indemnisations du fait qu'elle accorde une indemnisation partielle lorsque le degré d'appartenance du GPC à la classe concernée est inférieure à $1\left(\mu_{i}<1\right)$ et une indemnisation totale lorsque ce degré est égal à 1. La classification dure indemnise pour chaque classe indemnisable le noyau (Kern(A), voir définition 8) de la classe, tandis que la classification floue indemnise le support (Supp(A), voir définition 6). Une classe ou un ensemble flou indemnisable non vide $(A \neq \varnothing)$ mais non normalisée $(\operatorname{Kern}(\mathrm{A}) \neq \varnothing)$ n'accorde aucune indemnisation à ses éléments selon la classification dure alors que la classification floue indemnisera chacun de ses éléments en fonction de son degré d'appartenance à la classe. En indemnisant le support de façon proportionnelle au degré d'appartenance, la logique floue crée moins distorsion entre les GPC réduisant ainsi le risque base matérialisée par la différence entre le support et le noyau de la classe de risque considérée.

On peut augmenter la granularité de la classification floue en intégrant des variables sur les caractéristiques individuelles des GPC comme la taille du GPC, sa composition (rapport hommes/femmes), la technologie de production (utilisation de tracteur, de charrue ou de daba) etc. Ces informations peuvent être obtenues dans le cas de l'assurance rendement-coton du Burkina Faso sans coût additionnel à cause du suivi des GPC par les agents techniques de la Sofitex. Leur incorporation dans la matrice $\mathrm{X}$ fait tendre le contrat d'assurance indicielle vers un contrat individualisé c'est-à-dire des contrats "linéaires" proposant des versements d'indemnités plus continus. Le regroupement en catégorie des GPC peut tenir compte de ces différentes variables si l'objectif est la recherche d'une forte personnalisation des contrats. 
Une conséquence de la fuzzyfication est l'augmentation du montant des indemnisations due au fait que le support de l'ensemble flou (GPC concernés par l'indemnisation dans la classification floue) est toujours supérieur ou égal à son noyau (GPC concernés par l'indemnisation dans la classification dure $)(\operatorname{Supp}(\mathrm{A}) \geq \operatorname{Kern}(\mathrm{A}))$.

La fuzzyfication, en augmentant significativement la probabilité d'être indemnisé augmente également la prime. La prime actuarielle actuelle est de 6304 FCFA et la prime chargée de 11200 FCFA par hectare de coton, soit un taux de chargement de de $77,66 \%$ de la prime actuarielle. La prime actuarielle selon la classification floue dépend de la zone cotonnière, la moyenne nationale est 27000 FCFA par hectare. En considérant une appartenance minimale de $20 \%$ à la classe des indemnisables pour être indemnisé, cette prime moyenne est de l'ordre de 20000 FCFA. La fuzzyfication entraine une augmentation de la prime mais elle contribue à réduire significativement le risque de base. Elle pourrait donc contribuer à accroitre la demande d'assurance indicielle sous l'hypothèse que l'effet incitatif que produit le gain de justice dans l'indemnisation l'emporte sur l'effet désincitatif provoqué par l'augmentation de la prime. À cet effet, Elabed et Carter (2014a) ont découvert qu'environ deux tiers de producteurs de coton au Mali manifestaient une aversion à l'ambiguité ; ces producteurs seraient en principe prêts à payer des sommes substantielles pour atténuer ou éliminer totalement le risque de base.

\section{CONCLUSION}

L'assurance indicielle perçue par plusieurs analystes comme une solution prometteuse au problème du risque covariant pour les petits exploitants agricoles des pays en développement voit son impact fortement réduit par la mauvaise qualité de la plupart des contrats offerts dans ce domaine. Dans cet article nous considérons le contrat d'assurance indicielle rendement du coton au Burkina Faso pour examiner les implications de l'utilisation de la logique floue dans la classification des producteurs dans les différentes classes à indemniser. Nous avons montré que la classification floue qui autorise une appartenance partielle aux différentes classes et donc une indemnisation en fonction du degré d'appartenance à la classe augmente plus de trois fois la probabilité du producteur moyen d'être indemnisé. Cette forme d'indemnisation continue proportionnelle à la perte réduit le risque de base lié à l'hétérogénéité intra-groupe de l'indemnisation forfaitaire par grappe. Ainsi la classification floue contribue à rapprocher les pertes encourues 
par les producteurs aux indemnisations accordées par l'assurance ce qui pourrait relancer la demande de ce type d'assurance au sein des producteurs agricoles des pays en développement. La conséquence étroite de l'utilisation de la classification floue est l'augmentation de près de trois fois la prime actuarielle, toutefois des études (Elabed et Carter, 2014a) ont montré que les paysans sont prets à payer des sommes substantielles pour atténuer ou éliminer totalement le risque de base.

\section{BIBLIOGRAPHIE}

[1] Banque Mondiale (2015) Rapport sur le développement dans le monde: Nourrir l'Afrique de l'Ouest: Un agenda pour le commerce régional, Washington D.C.: Banque mondiale.

[2] Barré, T., Q. Stoeffler, and M. Carter (2016). Assessing index insurance: conceptual approach and empirical illustration from Burkina Faso.

[3] Barron J, Rockström J, Gichuki F, Hatibu N (2003) Dry spell analysis and maize yields for two semi-arid locations in east Africa. Agric For Meteorol 117: 23-37

[4] Bezdek, J.C. (1981), Pattern Recognition with Fuzzy Objective Function Algorithms, New York, Plenum Press.

[5] Carter M., de Janvry A., Sadoulet E. Sarris A. (2015), "Assurance climatique indicielle pour les pays en développement: examen des faits et propositions visant à augmenter le taux de souscription", Revue d'économie du développement (Vol. 23), p. 5-57. DOI 10.3917/edd.291.0005

[6] Chen, G, Lu.S and Yu, E (1980) Application of Fuzzy Set Theory to Economics In Advances in Fuzzy Set, Possibility Theory and Applications, (cd P. Wang) pp 227-305, Plenum Press, New York.

[7] Clarke, D. (2011a). "A theory of rational demand for index insurance", Série Documents de travail 572, département d'économie, Université d'Oxford

[8] Cummins, J.D., and Derrig, R.A. (1991), Fuzzy Financial Pricing of Property-Liability Insurance, working paper.

[9] Cummins, J.D., and Derrig, R.A. (1993), Fuzzy Trends in PropertyLiability Insurance Claim Costs, Journal of Risk ana' Insurance, forthcoming.

[10] Derrig RA, Ostaszewski KM (1999) Fuzzy sets methodologies in actuarial science. In : Zimmerman HJ (ed) Practical applications of fuzzy technologies. Kluwer Academic Publishers, Boston 
[11] DeWit, G.W. (1982), Underwriting andUncertainty, Insurance: Mathematics and Economics, $1: 277-285$.

[12] Ebanks B, Kanvowski W, Ostaszewski KM (1992) Application of measures of fuzziness to risk classification in insurance. In: Proceedings of the fourth international conference on computing and information: computing and information. IEEE Computer Society Press, Los Alamitos, California.

[13] Elabed, G. et M. Carter (2014a). "Basis Risk and Compound-risk Aversion: Evidence from a WTP Experiment in Mali ", Université de Californie à Davis

[14] Elabed, G., M. F. Bellemare, M. Carter, and C. Guirkinger (2013). Managing basis risk with multiscale index insurance. Agricultural Economics, 44(4-5): 419-431.

[15] Elabed, G.and M. Carter (2014). Ambiguity, compound-risk aversion and the demand for microinsurance. Technical report, University of California, Davis.

[16] Elabed, G.and M. Michael (2015) Ex-ante impacts of agricultural insurance: Evidence from a field experiment in mali.

[17] Erbach DW, Seah E (1993) Discussion of "The Application of Fuzzy Sets to Group Health Underwriting" by Young VR. Transactions of the Society of Actuaries 45: 585

[18] Graef F, Haigis J (2001) Spatial and temporal rainfall variability in the Sahel and its effects on farmers' management strategies. J Arid Environ 48: 221-231

[19] Kandel, A. (1982), Fuzzy Techniques in Pattern Recognition, John Wiley and Sons, New York.

[20] Lemiare, J. (1990), Fuzzy Insurance, Astin Bulletin, 20: 33-55.

[21] M. Mukaidono (2001). Fuzzy Logic for Beginners. World Scientific Publishing, London.

[22] Carter,M., G. Elabed, and E. Serfilippi (2015). Behavioral economic insights on index insurance design. Agricultural Finance Review, 75(1): 8-18.

[23] Ostaszewski K, Karwowski W (1992) An analysis of possible applications of fuzzy set theory to the actuarial credibility theory. In: Proceeding of the annual meeting of the North American fuzzy information processing society, Puerto Vallaria 
[24] Ostaszewski, K. (1993), An Investigation into Possible Applications of Fury Sets Methods in Actuarial Science, Society of Actuaries, Schaumburg, Illinois.

[25] OXFAM (2016), Rapport Etude d'impact assurance agricole, Rapport définitif mars 2016, Burkina Faso

[26] Planet Guarantee (2017), micro assurance en Afrique de l'Ouest expérience du Burkina, Planet Finance Group.

[27] Skees, J. (2008). «Innovations in Index Insurance for the Poor in Lower Income Countries ", Agricultural and Resource Economics Review 37: $1-15$.

[28] Verrall RJ, Yakoubov YH (1999) A fuzzy approach to grouping by policyholder age in general insurance. Journal of Actuarial Practice $7: 181-203$

[29] Vischel T, T. Lebel (2007) Assessing the water balance in the Sahel: impact of small scale rainfall variability on runoff. Part 2 : idealized modeling of runoff sensitivity. J Hydrol 333: 340-355

[30] Young VR (1993) The application of fuzzy sets to group health underwriting. Transactions of the Society of Actuaries 45: 551-590

[31] Zadeh L.A (1981) Fuzzy systems theory: a framework for the analysis of humanistic systems. In: Cavallo RE (ed) Recent developments in systems methodology in social science research. Kluwer, Boston : 25-41

[32] Zadeh, L.A. (1965), Fuzzy Sets, Information and Control, 8: 338-353

[33] Zadeh, L.A. (1965). Fuzzy sets. Information and Control, Vol. 8, pp. 338-353.

[34] Zadeh. L.A. (1978) Fuzzy Sets as a Basis for a Theory of Possibility. Fuzzy Sets and Systems, 1: 3-28.

[35] Zimmernuum, H.J. (1991), Fuzzy Set Theory and its Applications, Second Edition, Kluwer Academic Publishers, Boston, Massachusetts.

\section{NOTES}

1. Université Ouaga II, Burkina Faso, abel_tiemtore@yahoo.ca. 03 BP 7210 Ouagadougou 03

2. Barré et al. (2016) ont montré que le nombre de classes dans l'assurance-coton au Burkina Faso devrait être cinq pour obtenir une homogénéité intra-classe de $90 \%$ 